\title{
Distribution Design
}

\author{
Amos Beimel \\ Dept. of Computer Science \\ Ben Gurion University \\ amos.beimel@gmail.com \\ Yuval Ishai \\ Dept. of Computer Science \\ Technion and UCLA \\ yuvali@cs.technion.ac.il
}

\author{
Ariel Gabizon \\ Dept. of Computer Science \\ Technion \\ ariel.gabizon@gmail.com \\ Eyal Kushilevitz \\ Dept. of Computer Science \\ Technion \\ eyalk@cs.technion.ac.il
}

\begin{abstract}
Motivated by applications in cryptography, we introduce and study the problem of distribution design. The goal of distribution design is to find a joint distribution on $n$ random variables that satisfies a given set of constraints on the marginal distributions. Each constraint can either require that two sequences of variables be identically distributed or, alternatively, that the two sequences have disjoint supports. We present several positive and negative results on the existence and efficiency of solutions for a given set of constraints.

Distribution design can be seen as a strict generalization of several well-studied problems in cryptography. These include secret sharing, garbling schemes, and non-interactive protocols for secure multiparty computation. We further motivate the problem and our results by demonstrating their usefulness towards realizing non-interactive protocols for $a d-$ hoc secure multiparty computation, in which any subset of the parties may choose to participate and the identity of the participants should remain hidden to the extent possible.
\end{abstract}

\section{Keywords}

Secret sharing, secure multiparty computation, obfuscation, garbling schemes, multi-input functional encryption.

\section{INTRODUCTION}

Several questions in cryptography, including ones related to secret sharing $[13,4]$ and secure multiparty computation (MPC) $[16,8]$, call for the design of probability spaces with special properties. Such a probability space can often be described as a joint probability distribution $\left(X_{1}, X_{2}, \ldots, X_{n}\right)$ that satisfies a given set of constraints on the marginal distributions. Each constraint can either be an equality constraint of the form $\left(X_{i_{1}}, \ldots, X_{i_{d}}\right) \equiv\left(X_{i_{1}^{\prime}}, \ldots, X_{i_{d}^{\prime}}\right)$, in which case the two distributions should be identical, or a disjointness constraint of the form $\left(X_{i_{1}}, \ldots, X_{i_{d}}\right) \|\left(X_{i_{1}^{\prime}}, \ldots, X_{i_{d}^{\prime}}\right)$, in

Permission to make digital or hard copies of all or part of this work for personal or classroom use is granted without fee provided that copies are not made or distributed for profit or commercial advantage and that copies bear this notice and the full citation on the first page. Copyrights for components of this work owned by others than the author(s) must be honored. Abstracting with credit is permitted. To copy otherwise, or republish, to post on servers or to redistribute to lists, requires prior specific permission and/or a fee. Request permissions from Permissions@ acm.org. ITCS'16, January 14-16, 2016, Cambridge, MA, USA.

Copyright is held by the owner/author(s). Publication rights licensed to ACM. ACM 978-1-4503-4057-1/16/01 ...\$15.00.

DOI: http://dx.doi.org/10.1145/2840728.2840759 which case the two distributions should have disjoint supports. We will later consider a computational version of the problem in which equality is relaxed to computational indistinguishability.

As an example, consider the problem of sharing a secret $s \in\{0,1\}$ between $k$ parties, such that every $t$ parties learn nothing about the secret and every $t+1$ parties can reconstruct the secret from their shares. This problem can be formulated as that of designing a joint distribution on $n=2 k$ variables $X_{i}^{b}$, for $1 \leq i \leq k$ and $b \in\{0,1\}$, where $\left(X_{1}^{0}, \ldots, X_{k}^{0}\right)$ represent the shares of $s=0$ and $\left(X_{1}^{1}, \ldots, X_{k}^{1}\right)$ represent the shares of $s=1$. The secrecy requirement can be expressed by a set of equality constraints of the form $X_{T}^{0} \equiv X_{T}^{1}$ for all $T \subseteq[k]$ of size $t$. The reconstruction requirement can be expressed by a set of disjointness constraints of the form $X_{T}^{0} \| X_{T}^{1}$ for all $T \subseteq[k]$ of size $t+1$.

Other than secret sharing, several additional cryptographic primitives can be viewed as special cases of distribution design. These include: (1) $k$-party private simultaneous messages (PSM) protocols [5], which correspond to the special case where the variable set is partitioned into $k$ parts and each sequence of variables involves exactly one variable from each part; (2) information-theoretic garbling schemes [16, $10,3]$, which can be viewed as a special type of PSM protocols; (3) robust protocols for non-interactive secure multiparty computation (NIMPC) [2], an information-theoretic variant of multi-input functional encryption [9] that strengthens PSM protocols by imposing additional secrecy requirements; and (4) an information-theoretic variant of the notion of functional secret sharing from [11].

The above special cases only capture a small fragment of all distribution design instances that can be specified by a set of constraints. In fact, even general "graph-based" constraints, which only involve a pair of variables in each side, are not covered by any prior work we are aware of.

\subsection{Our Contribution}

We initiate a systematic study of the problem of distribution design. We address the following natural questions: (1) Which sets of constraints admit a solution? (2) What is the computational complexity of deciding whether a solution exists? (3) How efficient can the solutions be?

We give a simple answer to the first question: a set of constraints can be realized if and only if a natural closure of the equality constraints does not contradict any of the disjointness constraints. For the other questions we obtain 
partial answers, proving both positive and negative results. We now give a more detailed overview of the results.

We consider two special cases of the general problem that are of particular interest. A projective set of constraints is one where the constraints are restricted to be of the form $X_{S} \equiv X_{S^{\prime}}$ or $X_{S} \| X_{S^{\prime}}$ for sets $S, S^{\prime} \subset[n]$. That is, in such constraints each variable sequence is sorted according to the variable indices. For instance, $\left(X_{1}, X_{2}, X_{3}\right) \equiv\left(X_{2}, X_{3}, X_{1}\right)$ is a non-projective constraint. All of the above examples for distribution designs that arise in cryptography are projective. A d-homogenous set of constraints is one where all sequences have the same length $d$. Among the above examples, the constraints corresponding to PSM protocols and garbling schemes are homogenous, whereas those corresponding to secret sharing schemes and NIMPC protocols are not homogenous.

It will be convenient to describe the efficiency of our solutions by only referring to the share size, defined as the maximal bit-length of a variable (i.e., $\left.\max _{i}\left\lceil\log _{2}\left|\operatorname{support}\left(X_{i}\right)\right|\right\rceil\right)$ ), with the implicit understanding that the distribution can be sampled in time polynomial in this share size, the number of variables $n$, and the number of constraints $m$.

We obtain the following main results.

The projective case. In the case of projective constraints, we show that a solution exists if and only if the closure of the equality constraints defined by the following generation rules does not contradict any disjointness constraint. The generation rules include the natural symmetric and transitive rules (e.g., $X_{A} \equiv X_{B}$ and $X_{B} \equiv X_{C}$ generate $X_{A} \equiv X_{C}$ ) as well as the following projection rule: $X_{A} \equiv X_{B}$, where $A$ and $B$ are viewed as sequences of length $d$, generate $X_{A_{I}} \equiv X_{B_{I}}$ for every $I \subseteq[d]$. That is, one can remove from both sides of an equality constraint variables occurring in the same set of locations.

When the set of projective constraints is $d$-homogenous, this implies a simple polynomial-time algorithm for deciding whether a solution exists. In case it does exist, the share size of our construction is $O(m d \log n)$ bits. Our construction enjoys two other properties that are useful for applications: it is $d$-symmetric in the sense that the joint distribution of every $d$ variables is invariant under permutations, and it is $(d-1)$-secret in the sense that the joint distribution of every $d-1$ variables is the same. On the other hand, when the set of constraints is not homogenous, it is PSPACE-complete to decide whether a solution exists. In case it does exist, the share size of our construction is $O\left(2^{t} m n\right)$, where $t$ is an upper bound on the number of variables appearing in a single constraint. Thus, the non-homogenous case appears to be qualitatively harder (at least from a computational point of view) than the homogenous case.

The general case. The case of non-projective sets of constraints, where the variables may appear in different orders (e.g., $\left.\left(X_{1}, X_{2}, X_{3}\right) \equiv\left(X_{3}, X_{1}, X_{2}\right)\right)$, is somewhat more involved. First, as in the projective case, we can characterize the existence of solutions using a natural closure of the equality constraints. In addition to the generation rules mentioned above, we add the following additional rule: if $A, B$ are sequences of length $d$, then $X_{A} \equiv X_{B}$ generates $X_{\pi(A)} \equiv X_{\pi(B)}$ for every permutation $\pi:[d] \rightarrow[d]$.

If the $m$ constraints are $d$-homogenous then, as in the projective case, we show a polynomial-time algorithm for deciding whether a solution exists. However, in contrast to the projective case, here the share size of our construction is super-polynomial and is bounded by $n^{O\left(d^{2}\right)}$. For nonhomogenous sets of general constraints, we have a $2^{O(n)}$ time algorithm to decide whether a solution exists (as in the projective case, the decision problem is PSPACE-complete), and the share size of the construction is $n^{O\left(n^{2}\right)}$.

Implicitly represented constraints. Up to this point, we assumed that a set of $m$ constraints is given explicitly. However, for cryptographic applications it is often useful to consider an exponentially large number of constraints that are succinctly described by some implicit representation. More concretely, we assume that the constraints are implicitly represented by a circuit $C$ that, given a sequence of $n$ variable indices, either outputs a positive integer, representing an equivalence class, or 0, representing "don't care." (In the projective case, the input can be just an $n$-bit characteristic vector of a set.) If $C$ has the same nonzero output on two sequences, the corresponding distributions are required to be (either perfectly or computationally) indistinguishable, whereas if $C$ has different nonzero outputs the distributions should be efficiently distinguishable. In fact, we require the existence of an efficient decoder that recovers an identifier of the equivalence class given a sample from a distribution.

We present an efficient algorithm that, given such an implicit representation of a projective set of constraints, can efficiently sample from a distribution that satisfies the computational variant of the above requirements. The above is guaranteed to hold under a technical condition on $C$ that includes two interesting cases: the case where constraints are consistent and are fully specified (i.e., $C(S)$ never outputs 0$)$ and the case where the constraints are $d$-homogenous (i.e., $C(S)$ outputs 0 on all sets whose size is different from $d)$. The algorithm is based on the assumptions that indistinguishability obfuscation (iO) $[1,7]$ and one-way functions exist, and builds on recent constructions of multi-input functional encryption [9]. On the negative side, we also show that there is no similar algorithm in the general (non-projective) case, even for homogenous constraints, unless RP $=\mathrm{NP}$. This establishes a qualitative separation between the projective and the general case.

Application to secure ad-hoc computation. We further motivate the study of distribution design and our results by demonstrating their usefulness for realizing noninteractive protocols for ad-hoc secure multiparty computation, in which only a subset of the parties may choose to participate and the identity of participants should remain hidden to the extent possible. This application is an instance of distribution design which does not seem to be captured by known primitives. See Section 5 for more details.

Open questions. Many natural open questions concerning the complexity of distribution design are left open. For instance, can every set of constraints on $n$ variables be realized with share size that is polynomial in $n$ ? This question is wide open even in the special cases of secret sharing schemes and PSM protocols. However, the extra generality of distribution design may give rise to stronger lower bounds, and possibly serve as a stepping stone towards improved lower bounds for secret sharing and PSM protocols. A potentially easier question is that of obtaining sharp bounds on the minimal share size required by simple instances of distribution design, such as a design that "singles out" one set of $d$ variables (see Lemma 3.1). 


\section{PRELIMINARIES}

In this section we define the problem of distribution design and some of its useful special cases.

Notation 2.1. We denote by $[n]$ the set $\{1, \ldots, n\}$ and by $\langle n\rangle_{d}$ the set of ordered sequences of distinct elements from $[n]$ of length $d$; that is, an element of $\langle n\rangle_{d}$ has the form $\left(i_{1}, \ldots, i_{d}\right)$ where each $i_{j}$ is in $[n]$ and, for all $j_{1} \neq j_{2}$, we have $i_{j_{1}} \neq i_{j_{2}}$. Finally, let $\langle n\rangle \triangleq \cup_{d=1}^{n}\langle n\rangle_{d}$.

For a random variable $Y$ supported on a finite set $\operatorname{supp}(Y)$, denote $|Y|=\left\lceil\log _{2}|\operatorname{supp}(Y)|\right\rceil$. For a sequence of jointly distributed random variables $X=\left(X_{1}, \ldots, X_{n}\right)$ and a sequence of indices $S=\left(i_{1}, \cdots, i_{d}\right) \in\langle n\rangle$, we denote $X_{S} \triangleq$ $\left(X_{i_{1}}, \ldots, X_{i_{d}}\right)$.

For an (unordered) subset $A \subseteq[n]$, where $i_{1}<i_{2}<\cdots<$ $i_{d}$ are the elements of $A$ in ascending order, we identify the set $A$ with the (ordered) sequence $\left(i_{1}, \ldots, i_{d}\right)$, and denote $X_{A} \triangleq\left(X_{i_{1}} \ldots, X_{i_{d}}\right)$.

For two random variables $Y, Z$, we denote $Y \equiv Z$ if $Y$ and $Z$ are identically distributed, and $Y \| Z$ if the supports of $Y$ and $Z$ are disjoint.

Definition 2.2 (Distribution Design). A set of constraints $\mathcal{R}$ is a set of the form $\left\{\text { " } T_{i} \circ_{i} Q_{i}{ }^{\prime}\right\}_{i=1}^{m}$, where $T_{i}, Q_{i} \in$ $\langle n\rangle,\left|T_{i}\right|=\left|Q_{i}\right|$, and $\circ_{i} \in\{\equiv, \|\}$. A distribution design realizing $\mathcal{R}$ is a sequence $X=\left(X_{1}, \ldots, X_{n}\right)$ of random variables such that

- For every " $Q \equiv T " \in \mathcal{R}, X_{Q} \equiv X_{T}$.

- For every "Q\|T" $\in \mathcal{R}, X_{Q} \| X_{T}$.

Let $X=\left(X_{1}, \ldots, X_{n}\right)$ be a distribution design. Borrowing notation from secret sharing schemes, for $\left(x_{1}, \ldots, x_{n}\right) \in$ $\operatorname{supp}(X)$, we will call $x_{i}$ the ith share. We define the share size of $X$ to be $\max _{i \in[n]}\left|X_{i}\right|$.

A definition of computational distribution designs will be given in Section 6.1. Next, we consider the following special types of constraints, where the constraints are only on sets.

Definition 2.3 (Projective Set of COnstraints). The constraints in $\mathcal{R}$ are projective if for every " $S \circ Q$ " $\in \mathcal{R}$, where $S=\left(i_{1}, \ldots, i_{d}\right)$ and $Q=\left(j_{1}, \ldots, j_{d}\right)$, it holds that $i_{1}<i_{2}<\cdots<i_{d}$ and $j_{1}<j_{2}<\cdots<j_{d}$. We refer to distribution designs for such sets of constraints as being projective.

Note that projective constraints can be expressed by a set of constraints of the form $X_{S} \equiv X_{T}$ or $X_{S} \| X_{T}$ for sets $S, T$ of the same size.

DeFinition 2.4 (SYMmetric Distribution DESigns). A sequence of random variables $\left(Y_{1}, \ldots, Y_{d}\right)$ is symmetric if $\left(Y_{1}, \ldots, Y_{d}\right) \equiv\left(Y_{\pi(1)}, \ldots, Y_{\pi(d)}\right)$ for every permutation $\pi:[d] \rightarrow[d]$. A distribution design $X$ is $d$-symmetric if $X_{S}$ is symmetric for any $S \subseteq[n]$ with $|S|=d$.

Definition 2.5 ( $t$-SeCRet Distribution Designs). $A$ distribution design $X$ is t-secret if $X_{S} \equiv X_{Q}$ for any sequences $S, Q \in\langle n\rangle$ with $|Q|=|S| \leq t$.

Definition 2.6. A set of constraints $\mathcal{R}$ is $d$-homogenous if $|S|=|Q|=d$ for every constraint " $S \circ Q$ " $\in \mathcal{R}$. A set of constraints $\mathcal{R}$ is $t$-bounded if $|S|=|Q| \leq t$ for every constraint " $S \circ Q " \in \mathcal{R}$.
REMARK 2.7 (A NOTE ON EFFICIENCY). In all our constructions of distribution designs (as described in the various theorems throughout the paper), the running time of the algorithm for generating the distribution design is polynomial in the number of variables in the distribution design and in the share size.

Furthermore, when describing algorithms, we assume that for any integer $N$ the algorithm can perfectly sample a uniformly random number in the range $[N]$ in time poly $(\log N)$. If we insist on algorithms that can only generate random bits, some of our stated running times become expected rather than strict.

\section{PROJECTIVE DISTRIBUTION DESIGNS}

In this section we construct distribution designs for projective constraints. We start with homogenous projective sets of constraints, where we have a construction with polynomial share size; in the full version we deal with nonhomogenous projective sets of constraints. We only consider $d>1$ as the case $d=1$ is trivial (e.g., can be solved with one vector of shares). We construct a distribution design for homogeneous constraints in three steps. In the first step, we present a construction of a distribution design that "singles out" one set $A_{0}$ of size $d$.

Lemma 3.1. Let $A_{0} \subseteq[n]$ be a set of size $1<d<n$ and consider the $d$-homogenous projective set of constraints $\mathcal{R}_{A_{0}}=\left\{\right.$ " $A \| A_{0}$ " : $\left.A \subset[n],|A|=d, A \neq A_{0}\right\} \cup\left\{\right.$ " $A \equiv A^{\prime}$ " : $\left.A, A^{\prime} \subset[n],|A|=\left|A^{\prime}\right|=d, A, A^{\prime} \neq A_{0}\right\}$. Then, there exists $a(d-1)$-secret d-symmetric distribution design for $\mathcal{R}_{A_{0}}$ with share size at most $\min \{2 d \cdot \log n, n-1\}$.

Proof. For simplicity of notation, assume that $A_{0}=$ $\{1, \ldots, d\}$. We first describe, slightly informally, the solution with share size $n-1$ : Choose $X_{2}, \ldots, X_{n}$ to be random linearly independent vectors in $\mathbb{F}_{2}^{n-1}$, set $X_{1} \triangleq \sum_{i=2}^{d} X_{i}$ and let $X^{\mathrm{I}}=\left(X_{1}, \ldots, X_{n}\right)$. It is easy to see that any set of size $d$ besides $A_{0}$ "sees" $d$ random linearly independent vectors, whereas $X_{A_{0}}^{\mathrm{I}}$ consists of $d-1$ random linearly independent vectors followed by their sum.

We now describe the solution with share size $2 d \cdot \log n$. Let $\mathbb{F}$ be a field of characteristic two of size $n \leq|\mathbb{F}| \leq 2 n$. Fix vectors $v_{2}, \ldots, v_{n} \in \mathbb{F}^{2 d-1}$ that are $2 d-1$-wise linearly independent, i.e., for any sequence $1<i_{1}<\cdots<i_{2 d-1} \leq n$, the vectors $v_{i_{1}}, \ldots, v_{i_{2 d-1}}$ are linearly independent over $\mathbb{F}$ (using Vandermonde matrices, it is well-known that such a set of vectors exists when $|\mathbb{F}| \geq n-1)$. Let $v_{1} \triangleq \sum_{i=2}^{d} v_{i}$. We have the following relations between $v_{1}, \ldots, v_{n}$.

- $\sum_{i=1}^{d} v_{i}=0$.

- If $S \subsetneq\{1, \ldots, d\}$, the vectors $\left\{v_{i}\right\}_{i \in S}$ are linearly independent.

- If $S=\left\{i_{1}, \ldots, i_{d}\right\} \neq\{1, \ldots, d\}$ then $v_{i_{1}}, \ldots, v_{i_{d}}$ are linearly independent: If $1 \notin S$, this is immediate. When $1 \in S$, if the vectors $\left\{v_{i}\right\}_{i \in S}$ are dependent, then the set of vectors $\left\{v_{2}, \ldots, v_{d}\right\} \cup\left\{v_{i}\right\}_{i \in S \backslash\{1\}}$ of size at most $2 d-1$ are dependent, contradicting the choice of $v_{2}, \ldots, v_{n}$.

Denote $s \triangleq 2 d-1$. Define a sequence of random variables $X^{\mathrm{II}}=\left(X_{1}, \ldots, X_{n}\right)$ as follows. Uniformly select a random $\mathbb{F}$-linear map $T: \mathbb{F}^{s} \rightarrow \mathbb{F}^{s}$ of full rank, i.e., $T\left(\mathbb{F}^{s}\right)=\mathbb{F}^{s}$. Now, for $i \in[n]$, define $X_{i} \triangleq T\left(v_{i}\right)$. We claim that $X^{\mathrm{II}}$ 
realizes $\mathcal{R}_{A_{0}}$ : fix any subset $A \neq A_{0}$ of $[n]$ of size $d$. The set of vectors $\left\{v_{i}\right\}_{i \in A}$ is linearly independent. As one way to sample $T$ is by first choosing random linearly independent vectors $\left\{T\left(v_{i}\right)\right\}_{i \in A}$ and then defining $T$ on some completion of $\left\{v_{i}\right\}_{i \in A}$ to a basis, $X_{A}^{\mathrm{II}}=\left(T\left(v_{i}\right)\right)_{i \in A}$ is uniformly distributed amongst sequences of $d$ linearly independent vectors in $\mathbb{F}^{s}$. In particular, $X_{A}^{\mathrm{II}} \equiv X_{B}^{\mathrm{II}}$ for any $A, B \neq A_{0}$ of size $d$. Furthermore, $X_{A}^{\mathrm{II}}$ is a symmetric distribution, as a random permutation of $d$ random linearly independent vectors is itself a sequence of $d$ random linearly independent vectors.

On the other hand, any sequence $\left(u_{1}=T\left(v_{1}\right), \ldots, u_{d}=\right.$ $\left.T\left(v_{d}\right)\right)$ in the support of $X_{A_{0}}^{\mathrm{II}}$ will satisfy $\sum_{i=1}^{d} u_{i}=0$. In particular, there will not be any sequences of $d$ linearly independent vectors in the support of $X_{A_{0}}$ and hence, $X_{A_{0}}^{\mathrm{II}} \| X_{A}^{\mathrm{II}}$ for any $A \neq A_{0}$. Furthermore, any $d-1$ vectors out of $\left\{u_{1}, \ldots, u_{d}\right\}$ are random linearly independent vectors. Thus, $X_{A_{0}}^{\mathrm{II}}$ is also a symmetric distribution.

Using the above lemma, we can "separate" one set $\mathcal{A}$ of subsets of size $d$ from all other subsets of size $d$.

Lemma 3.2. Given a set $\mathcal{A}$ of subsets of $[n]$ of size $d$, there is a $(d-1)$-secret $d$-symmetric distribution design $X$ of share size $|\mathcal{A}| \cdot \min \{2 d \cdot \log n, n-1\}$ such that for any $A, B \subseteq[n]$ of size $d$

- If $A, B \in \mathcal{A}$ or $A, B \notin \mathcal{A}$, then $X_{A} \equiv X_{B}$.

- If $A \in \mathcal{A}$ and $B \notin \mathcal{A}$, then $X_{A} \| X_{B}$.

Proof. Let $s=|\mathcal{A}|$ and denote $\mathcal{A}=\left\{A_{1}, \ldots, A_{s}\right\}$. For each $i \in[s]$, we use Lemma 3.1 to generate a distribution $\operatorname{design} X^{i}=\left(X_{1}^{i}, \ldots, X_{n}^{i}\right)$ of share size $\min \{2 d \cdot \log n, n-1\}$, such that $X_{A_{i}} \| X_{B}$ for all $A_{i} \neq B \subseteq[n]$ with $|B|=d$. We next choose a random permutation $\pi$ of $[s]$. Now, we define a distribution design $X=\left(X_{1}, \ldots, X_{n}\right)$ by $X_{j} \triangleq\left(X_{j}^{\pi(i)}\right)_{i \in[s]}$. That is, for each set $A_{0} \in A$, we generate shares according to the distribution design of Lemma 3.1, and we concatenate these shares in a random order.

We next argue that this distribution design satisfies all the constraints. First fix a set $A \in \mathcal{A}$. We view the random variable $X$ as consisting of $s$ rows, each row corresponding to shares generated according to one copy of the distribution design of Lemma 3.1 and consider $X_{A}$ - these rows restricted to $A$. Of these restricted rows, $s-1$ rows contain $d$ random linearly independent vectors, and the remaining row, whose location is uniformly distributed because of $\pi$, contains $d-1$ random linearly independent vectors followed by their sum. For a set $B \notin \mathcal{A}$, the random variable $X_{B}$ simply consists of $s$ rows each containing $d$ random linearly independent vectors. The lemma follows.

Before proceeding to the main result of this subsection, we introduce the notion of concatenation of distribution designs: Given distribution designs $X^{1}=\left(X_{1}^{1}, \ldots, X_{n}^{1}\right)$ and $X^{2}=\left(X_{1}^{2}, \ldots, X_{n}^{2}\right)$, we define the distribution design $X=$ $X^{1} \circ X^{2}$ by $X=\left(X_{1}, \ldots, X_{n}\right)$ where $X_{i} \triangleq X_{i}^{1} \circ X_{i}^{2}$. For sets $A, B \subseteq[n]$, the following hold:

- If $X_{A}^{1} \equiv X_{B}^{1}$ and $X_{A}^{2} \equiv X_{B}^{2}$, then $X_{A} \equiv X_{B}$.

- If $X_{A}^{1} \| X_{B}^{1}$ or $X_{A}^{2} \| X_{B}^{2}$, then $X_{A} \| X_{B}$.

We note that the distribution designs of Lemmas 3.1 and 3.2 can clearly be sampled in polynomial time in $n$ and their share sizes. This will be used in the following theorem.
THEOREM 3.3. There exists an algorithm running in time poly $(|\mathcal{R}|, n)$ that given a projective d-homogenous set of constraints $\mathcal{R}$ on $n$ variables

- determines whether $\mathcal{R}$ is realizable.

- If so, returns a sample $\left(x_{1}, \ldots, x_{n}\right)$ from $a(d-1)$ secret d-symmetric distribution design $X$ realizing $\mathcal{R}$ with share size $2|\mathcal{R}| \cdot \min \{2 d \cdot \log n, n-1\}$.

Proof. The algorithm starts by computing the set of equivalence classes of sets of size $d$ "induced" by $\mathcal{R}$. More precisely, we create a graph of the subsets of size $d$ appearing in one of the constraints in $\mathcal{R}$. We connect vertices $A$ and $B$ if and only if " $A \equiv B$ " $\in \mathcal{R}$, and find the connected components $\mathcal{A}_{1}, \ldots, \mathcal{A}_{\ell}$ of this graph. Note that the total number of vertices in the graph is at most $2|\mathcal{R}|$. We now check whether for some constraint " $A \| B$ " $\in \mathcal{R}$ the sets $A$ and $B$ are in the same component $\mathcal{A}_{i}$. If so, we declare that $\mathcal{R}$ is not realizable. Otherwise, " $A \equiv B$ " $\in \mathcal{R}$ obviously implies that $A$ and $B$ are in the same connected component, and " $A \| B$ " $\in \mathcal{R}$ implies that $A$ and $B$ are in different components. Thus, it is enough to construct a distribution design $X$ where $X_{A} \equiv X_{B}$ whenever $A$ and $B$ are in the same component $\mathcal{A}_{i}$, and $X_{A} \| X_{B}$ whenever $A$ and $B$ are in different components. First, for each class $\mathcal{A}_{i}$ we generate a distribution design $X^{i}$ separating $\mathcal{A}_{i}$ from all other subsets of size $d$ using Lemma 3.2. The share size will be $\left|\mathcal{A}_{i}\right| \cdot \min \{2 d \cdot \log n, n-1\}$. Now define the distribution de$\operatorname{sign} X \triangleq X^{1} \circ \cdots \circ X^{\ell}$. On one hand, if " $A \equiv B$ " $\in \mathcal{R}$ for two sets $A, B$, then $A, B \in \mathcal{A}_{i}$ for some $i \in[\ell]$, and $X_{A}^{j} \equiv X_{B}^{j}$ for all $j \in[\ell]$, thus, $X_{A} \equiv X_{B}$. On the other hand, if " $A \| B$ " $\in \mathcal{R}$ for two sets $A, B$, then $A \in \mathcal{A}_{i}, B \in \mathcal{A}_{j}$ for $i \neq j$, thus, $X_{A}^{i} \| X_{B}^{i}$, implying that $X_{A} \| X_{B}$. Therefore, $X$ realizes $\mathcal{R}$. The claim regarding share size follows from the fact that $\left|\mathcal{A}_{1}\right|+\cdots+\left|\mathcal{A}_{\ell}\right| \leq 2|\mathcal{R}|$.

In the full version of this paper, we present a construction of distribution designs realizing non-homogenous projective sets of constraints. The idea of the construction is to partition the set of constraints $\mathcal{R}$ into sets $\mathcal{R}_{1}, \ldots, \mathcal{R}_{n}$, where $\mathcal{R}_{d}$ contains all constraints in $\mathcal{R}$ with sets of size $d$. We would like to realize each $\mathcal{R}_{d}$ independently. However, we need to add constraints to ensure the consistency. For example, if " $\{1,2,3\} \equiv\{4,5,6\} " \in \mathcal{R}$, then we will add the constraint " $\{1,3\} \equiv\{4,6\} "$ (and all other constraints with sets of size 1 and 2 for the appropriate subsets). After adding these additional constraints we can realize each $\mathcal{R}_{d}$ and concatenate the shares; using additional properties of the distribution design we constructed (e.g., $(d-1)$-secrecy), we can prove that the resulting design realizes $\mathcal{R}$.

\section{CONSTRUCTIONS OF GENERAL DIS- TRIBUTION DESIGNS}

In this section, we characterize when a set of constraints $\mathcal{R}$ can be realized by a distribution design. For lack of space, most of the proofs in this section are deferred to the full version of the paper.

\subsection{A Construction for $n$-Homogeneous Sets of Constraints}

As a first step of the characterization, we consider an $n$ homogeneous set of constraints, that is, all the sequences 
appearing in the constraints are of length $n$, i.e., each sequence is a permutation. In the following, we represent a permutation $\pi \in S_{n}$ by a vector $(\pi(1), \ldots, \pi(n))$ (this notation should not be confused with describing a permutation as a list of cycles). The latter vector is also used as a vector of shares in a distribution design.

We next recall some background on $S_{n}$, the group of permutations over $[n]$. For two permutations $\pi_{1}, \pi_{2} \in S_{n}$, let $\pi=\pi_{1} \cdot \pi_{2}$ be the permutation defined by $\pi(a)=\pi_{2}\left(\pi_{1}(a)\right)$ for every $a \in[n]$. Let $G$ be a subgroup of $S_{n}$. A left coset of $G$ is the set of permutations $\left\{\pi_{0} \cdot \pi: \pi \in G\right\}$ for some $\pi_{0} \in S_{n}$. The left cosets of $G$ partition $S_{n}$ into disjoint cosets (of equal size). Two permutations $\pi_{1}, \pi_{2} \in S_{n}$ are in the same left coset of $G$ if and only if $\pi_{1}^{-1} \cdot \pi_{2} \in G$. Finally, let id be the identity permutation.

Clearly, if $\mathcal{R}$ contains the constraints " $S \equiv Q$ ", " $Q \equiv$ $T$ ", "S\|T", then $\mathcal{R}$ cannot be realized by a distribution design. However, there are more complex reasons why a set of constraints $\mathcal{R}$ cannot be realized. The next example shows such $\mathcal{R}$ and gives a motivation for using the group of permutations.

EXAmple 4.1. Consider the set of constraints $\mathcal{R}_{\text {swap }}$ that contains the constraint $(1, \ldots, n) \equiv(1, \ldots, i-1, i+1, i, i+$ $2, \ldots, n)$ for every $1 \leq i \leq n-1$. Notice that $(1, \ldots, i-$ $1, i+1, i, i+2, \ldots, n)$ is the permutation that swaps the $i$-th and $(i+1)$-th elements. Assume that there is a distribution design $X$ realizing $\mathcal{R}_{\text {swap }}$ and let $\left(x_{1}, \ldots, x_{n}\right)$ be any vector of shares in the support of $X$. Since $(1, \ldots, n) \equiv$ $(2,1,3, \ldots, n)$ we get that $\left(x_{2}, x_{1}, x_{3}, \ldots, x_{n}\right)$ is in the support of $X$. Since $(1, \ldots, n) \equiv(1,3,2,4, \ldots, n)$ we get that also $\left(x_{2}, x_{3}, x_{1}, x_{4}, \ldots, x_{n}\right)$. We can apply such steps (at most $O\left(n^{2}\right)$ times) and conclude that every permutation of $\left(x_{1}, \ldots, x_{n}\right)$ is in the support of $X$, thus, $X \equiv X_{S}$ for every permutation $S$.

We have shown that if $X_{\mathrm{id}} \equiv X_{S}$, for some permutation $S$, then $X_{\mathrm{id}} \equiv X_{S^{\prime}}$, where the permutation $S^{\prime}$ is obtained from $S$ by swapping the $i$-th and $(i+1)$-th elements. In other words, we consider the group of permutations generated by swaps and all of them are equivalent. As swaps generate all permutations, then in any distribution design realizing $\mathcal{R}_{\text {swap }}$ all permutations are equivalent.

We characterize the $n$-homogeneous sets of constraints that can be realized by a distribution design (using groups terminology).

TheOrem 4.2. Let $\mathcal{R}$ be an n-homogeneous set of constraints and let $G$ be the subgroup of $S_{n}$ generated by the permutations $\left\{Q^{-1} \cdot S: " S \equiv Q " \in \mathcal{R}\right\}$. Then, there exists a distribution design realizing $\mathcal{R}$ if and only if for every constraint "S\|Q" $\in \mathcal{R}$ the permutations $S$ and $Q$ are in different left cosets of $G$.

The theorem follows from the following two lemmas.

LEMMA 4.3. Let $\mathcal{R}$ be an n-homogeneous set of constraints and let $G$ be the subgroup of $S_{n}$ generated by the permutations $\left\{Q^{-1} \cdot S: " S \equiv Q\right.$ " $\left.\in \mathcal{R}\right\}$. If for every "S\|Q" $\in \mathcal{R}$ the permutations $S$ and $Q$ are in different left cosets of $G$, then there exists a distribution design realizing $\mathcal{R}$.

Proof. Consider the following distribution design: Choose, uniformly at random, a permutation $\pi \in G$, and let $X_{i}=$ $\pi(i)$ for $i \in[n]$. We claim that $X=\left(X_{1}, \ldots, X_{n}\right)$ is a distribution design for $\mathcal{R}$. Note that any vector of shares of $X$ is, by the construction, a permutation. Furthermore, for any sequence $S$ of length $n$, a permutation $\tau$ is in $\operatorname{supp}\left(X_{S}\right)$ if and only if there is a permutation $\pi \in \operatorname{supp}(X)$, i.e. $\pi \in G$, such that $\tau=S \cdot \pi$.

We next prove that $X$ satisfies each constraint in $\mathcal{R}$. First, assume that " $S \equiv Q$ " $\in \mathcal{R}$, and let $\tau \in \operatorname{supp}\left(X_{S}\right)$. Thus, there is a permutation $\pi \in \operatorname{supp}(X)=G$ such that $\pi=$ $S^{-1} \cdot \tau$. As $Q^{-1} \cdot S$ is a generator of $G$, we deduce that $\left(Q^{-1} \cdot S\right) \cdot \pi \in G$. Therefore, $\left(Q^{-1} \cdot S\right) \cdot \pi=\left(Q^{-1} \cdot S\right)$. $\left(S^{-1} \cdot \tau\right)=Q^{-1} \cdot \tau \in G=\operatorname{supp}(X)$. We conclude that $\tau=$ $Q \cdot\left(Q^{-1} \cdot \tau\right) \in \operatorname{supp}\left(X_{Q}\right)$. We have proved that $\operatorname{supp}\left(X_{S}\right)=$ $\operatorname{supp}\left(X_{Q}\right)$. As the vectors of shares $\pi$ and $Q^{-1} \cdot S \cdot \pi$ are chosen with equal probability, i.e. $1 /|G|$, the variables $X_{S}$ and $X_{Q}$ are equally distributed.

Next, assume that " $S \| Q$ " $\in \mathcal{R}$, and let $\tau \in \operatorname{supp}\left(X_{S}\right)$. There is a permutation $\pi \in \operatorname{supp}(X)=G$ such that $\pi=$ $S^{-1} \cdot \tau$, which implies $S=\tau \cdot \pi^{-1}$. If $\tau$ is also in $\operatorname{supp}\left(X_{Q}\right)$, then there is a permutation $\pi^{\prime} \in \operatorname{supp}(X)=G$ such that $\pi^{\prime}=Q^{-1} \cdot \tau$, which implies $Q=\tau \cdot\left(\pi^{\prime}\right)^{-1}$. Thus, $S$ and $Q$ are in the same left coset of $G$ (specifically, $\tau \cdot G$ ), contradicting the assumptions of the lemma. This implies that $\operatorname{supp}\left(X_{S}\right)$ and $\operatorname{supp}\left(X_{Q}\right)$ are disjoint, as required.

LEMMA 4.4. Let $\mathcal{R}$ be an $n$-homogeneous set of constraints and let $G$ be the subgroup of $S_{n}$ generated by the permutations $\left\{Q^{-1} \cdot S: " S \equiv Q " \in \mathcal{R}\right\}$. If there exists a distribution design realizing $\mathcal{R}$, then for every "S\|Q" $\in \mathcal{R}$ the permutations $S$ and $Q$ are in different left cosets of $G$.

Proof Sketch. Let $\left(x_{1}, \ldots, x_{n}\right)$ be a vector of shares in $\operatorname{supp}(X)$. It can be proved that $\left(x_{\pi(1)}, \ldots, x_{\pi(n)}\right) \in \operatorname{supp}(X)$, for every $\pi \in G$ (this is proved by induction on the number of steps by which $\pi$ is generated). Next, assume that $S$ and $Q$ are in the same left coset of $G$ and let $\left(x_{1}, \ldots, x_{n}\right)$ be a vector in the support of $X$. On one hand, $\left(x_{S(1)}, \ldots, x_{S(n)}\right) \in$ $\operatorname{supp}\left(X_{S}\right)$. On the other hand, $Q^{-1} \cdot S \in G$ since $S$ and $Q$ are in the same left coset of $G$. Thus, by the above induction, $\left(x_{\left(Q^{-1} \cdot S\right)(1)}, \ldots, x_{\left(Q^{-1} \cdot S\right)(n)}\right) \in \operatorname{supp}(X)$ and the vector $\left(x_{Q \cdot\left(Q^{-1} \cdot S\right)(1)}, \ldots, x_{Q \cdot\left(Q^{-1} \cdot S\right)(n)}\right)=\left(x_{S(1)}, \ldots, x_{S(n)}\right)$ is in $\operatorname{supp}\left(X_{Q}\right)$. Therefore, there is a vector of shares in both $\operatorname{supp}\left(X_{S}\right)$ and $\operatorname{supp}\left(X_{Q}\right)$ implying that " $S \| Q$ " $\notin \mathcal{R}$.

We next summarize our results for $n$-homogeneous sets of constraints.

THEOREM 4.5. There exists an algorithm running in time $\operatorname{poly}(n,|\mathcal{R}|)$ that given an $n$-homogeneous set of constraints $\mathcal{R}$ on $n$ variables,

- determines whether $\mathcal{R}$ is realizable.

- If so, returns a sample $\left(x_{1}, \ldots, x_{n}\right)$ from a distribution design $X$ realizing $\mathcal{R}$ with share size $\log _{2} n$.

Proof. By Theorem 4.2, we need to check that $S, Q$ are in different left cosets of $G$ for every " $S \| Q$ " $\in \mathcal{R}$. Checking whether two permutations $S, Q$ are in the same left coset of $G$ is equivalent to checking whether $S^{-1} Q$ is in $G$, which, when $G$ is given as a list of generators, can be computed in polynomial time in the number of generators and in $n$ (see, e.g., $[14,6]$ ). If $\mathcal{R}$ is realizable, the distribution design of Lemma 4.3 , which realizes $\mathcal{R}$, samples a permutation in $\pi \in G$ with uniform distribution and sets $X_{i}=\pi(i)$. Thus, the share size of the distribution design is $\log n$. Furthermore, there is a randomized algorithm running in time 
polynomial in $n$ and the number of generators of $G$ that, given a set of generators of a subgroup of $S_{n}$, outputs a random permutation in the group with uniform distribution (see, e.g., [12, Page 30]).

\subsection{A Construction for Homogeneous Sets of Constraints}

As the next step of constructing distribution designs, we characterize when $d$-homogeneous sets of constraints can be realized for some $d<n$.

Notation 4.6. Given a sequence $S=\left(i_{1}, \ldots, i_{d}\right) \in\langle n\rangle_{d}$ and a permutation $\pi \in S_{d}$, denote $\pi(S)=\left(i_{\pi(1)}, \ldots, i_{\pi(d)}\right)$.

Given a matrix $M$ with $n$ columns, and a sequence $S=$ $\left(i_{1}, \ldots, i_{d}\right)$ we define the matrix $M l_{S}$ as the restriction of $M$ to the columns indexed by $S$, that is, the $j$-th column of $M \iota_{S}$ is the $i_{j}$-th column of $M$.

Assume that there is a distribution design $X$ realizing $\mathcal{R}$. For every sequence $S \in\langle n\rangle$, the distribution design $X_{S}$ satisfies all constraints in $\mathcal{R}$ involving only permutations of $S$. This implies that if $X$ realizes $\mathcal{R}$, then $\mathcal{R}$ restricted to $S$ has to satisfy the conditions of Theorem 4.2. Furthermore,

OBSERVATION 4.7. Let $X$ be a distribution design realizing a set of constraints $\mathcal{R}$. For every two sequences $S, T \in$ $\langle n\rangle_{d}$, if "S$\equiv T$ " $\in \mathcal{R}$ and $" T \equiv \pi(T) " \in \mathcal{R}$ then $X_{S} \equiv X_{\pi(S)}$.

As a warmup, we consider 2-homogeneous sets of constraints with exactly two equivalence classes (and an additional restriction described bellow). This construction contains the ideas of the construction for arbitrary $d$-homogenous sets of constrains.

Formally, we consider a set of constraints $\mathcal{R}$ for which there are two sequences $T_{1}, T_{2} \in\langle n\rangle_{2}$ such that " $T_{1} \| T_{2}$ " $\in \mathcal{R}$ and for every $T \in\langle n\rangle_{2}$ it holds that " $T \equiv T_{\alpha}$ " $\in \mathcal{R}$ for exactly one $\alpha \in\{1,2\}$. We extend $\mathcal{R}$ to $\mathcal{R}^{\prime}$ by considering the transitive closure of $\equiv$, that is, $\mathcal{R}^{\prime}$ contains $\mathcal{R}$ and for every $\alpha, S_{1}, S_{2}$ such that " $S_{1} \equiv T_{\alpha}$ " $\in \mathcal{R}$ and " $S_{2} \equiv T_{\alpha}$ " $\in \mathcal{R}$, we add " $S_{1} \equiv S_{2}$ " $\in \mathcal{R}^{\prime}$. Clearly, if a distribution design realizes $\mathcal{R}$, then it realizes $\mathcal{R}^{\prime}$. We define a function $f:\langle n\rangle_{2} \rightarrow\{1,2\}$, where $f\left(i_{1}, i_{2}\right)=\alpha$ for the $\alpha$ such that " $\left(i_{1}, i_{2}\right) \equiv T_{\alpha}$ " $\in \mathcal{R}$. Assume that $X$ realizes $\mathcal{R}$. Then, for every $S_{1}, S_{2} \in\langle n\rangle_{2}$, if $f\left(S_{1}\right)=f\left(S_{2}\right)$ then $X_{S_{1}} \equiv X_{S_{2}}$, otherwise, $X_{S_{1}} \| X_{S_{2}}$.

Assume that $f\left(j_{1}, j_{2}\right) \neq f\left(j_{2}, j_{1}\right)$ for every $j_{1} \neq j_{2}$. We next show that, in this case, $\mathcal{R}$ can be realized by a distribution design. Consider the following matrix $N$ with $n$ columns. For every pair $\left(i_{1}, i_{2}\right) \in[n]^{2}$, where $i_{1}<i_{2}$, we have a row in the matrix, where the $i_{1}$-th entry is $f\left(i_{1}, i_{2}\right)$ and the $i_{2}$-nd entry is $f\left(i_{2}, i_{1}\right)$; all other entries in this row are 0 . We next add rows to $N$. For every $1 \leq i \leq n$, let $\#_{i, 1}=\left|\left\{\left(i, i_{2}\right): i<i_{2}, f\left(i, i_{2}\right)=1\right\}\right|+\mid\left\{\left(i_{1}, i\right): i>\right.$ $\left.i_{1}, f\left(i_{1}, i\right)=1\right\} \mid$. That is, $\#_{i, 1}$ is the number of entries that are 1 in the $i$-th column and $n-1-\#_{i, 1}$ is the number of entries that are 2 in this column. We add $n-1-\#_{i, 1}$ rows to the matrix, where the $i$-th entry is 1 ; all other entries in these rows are 0 . Similarly, we add $\#_{i, 1}$ rows to the matrix, where the $i$-th entry is 2 ; all other entries in these rows are 0 . Thus, the number of 1's and 2's in each column is $n-1$.

Now consider $\left.N\right|_{\left\{i_{1}, i_{2}\right\}}$, the matrix $N$ restricted to columns $i_{1}$ and $i_{2}$ for some $i_{1}<i_{2}$. All rows in $N L_{\left\{i_{1}, i_{2}\right\}}$, except for one, contain at most 1 non-zero entry. In the row where there are two non-zero elements (the row labeled by $\left(i_{1}, i_{2}\right)$ ), if $f\left(i_{1}, i_{2}\right)=1$ then the entries are $(1,2)$, otherwise the entries are $(2,1)$. This implies that for every $i_{1}, i_{2}$ such that $f\left(i_{1}, i_{2}\right)=1$, the matrix $\left.N\right|_{\left\{i_{1}, i_{2}\right\}}$ is the same up to a permutation of the rows. This is also true for all matrices $\left.N\right|_{\left\{i_{1}, i_{2}\right\}}$ for $i_{1}, i_{2}$ such that $f\left(i_{1}, i_{2}\right)=2$. Thus, to construct a distribution design realizing $\mathcal{R}$, we choose with uniform distribution a permutation on the rows of the matrix, and the $i$-th share is the $i$-th permuted row.

ExAmple 4.8. Consider the 2-homogeneous set of requirements $\mathcal{R}$ with 3 variables and two equivalence classes, described by the constraints " $(1,2) \|(2,1)$ ", " $(1,2) \equiv(1,3)$ ", $"(1,2) \equiv(3,2) ", "(2,1) \equiv(3,1) ", "(2,1) \equiv(2,3)$. To construct a distribution design realizing $\mathcal{R}$, we first construct the following matrix.

\begin{tabular}{c|ccc|} 
& 1 & 2 & 3 \\
\hline$(1,2)$ & 1 & 2 & 0 \\
$(1,3)$ & 1 & 0 & 2 \\
$(2,3)$ & 0 & 2 & 1
\end{tabular}

In this matrix $\#_{1,1}=2, \#_{2,1}=0$, and $\#_{3,1}=1$. So we add rows to the matrix and obtain $N$ :

\begin{tabular}{c|ccc} 
& 1 & 2 & 3 \\
\hline$(1,2)$ & 1 & 2 & 0 \\
$(1,3)$ & 1 & 0 & 2 \\
$(2,3)$ & 0 & 2 & 1 \\
& 2 & 0 & 0 \\
& 2 & 0 & 0 \\
& 0 & 1 & 0 \\
& 0 & 1 & 0 \\
& 0 & 0 & 1 \\
& 0 & 0 & 2
\end{tabular} \mid

To construct a distribution design realizing $\mathcal{R}$, we randomly permute the rows of $N$ and $X_{i}$ is the permuted $i$-th column of $N$.

As we have seen in Theorem 4.7, constraints on a pair of sequences imply constraints on other sequences. This motivates the definition of $\operatorname{cl}(\mathcal{R})$ - the closure of the set of constraints $\mathcal{R}$ - which contains all constraints implied by $\mathcal{R}$.

DEFINITION 4.9. Let $\mathcal{R}$ be a d-homogeneous set of constraints. The closure of $\mathcal{R}$, denoted $\operatorname{cl}(\mathcal{R})$, is the minimal set such that:

1. $\mathcal{R} \subseteq \operatorname{cl}(\mathcal{R})$,

2. If " $S \equiv Q " \in \operatorname{cl}(\mathcal{R})$, then " $Q \equiv S " \in \operatorname{cl}(\mathcal{R})$,

3. If " $S \circ Q " \in \operatorname{cl}(\mathcal{R})$ for some $\circ \in\{\equiv, \|\}$, then " $S \equiv S " \in$ $\operatorname{cl}(\mathcal{R})$,

4. If " $S \equiv Q$ " $\in \operatorname{cl}(\mathcal{R})$ and " $Q \circ T$ " $\in \operatorname{cl}(\mathcal{R})$ for some $\circ \in\{\equiv, \|\}$, then " $S \circ T$ " $\in \operatorname{cl}(\mathcal{R})$,

5. If " $S \circ Q$ " $\in \operatorname{cl}(\mathcal{R})$, then " $\pi(S) \circ \pi(Q)$ " $\in \operatorname{cl}(\mathcal{R})$ for every $\pi \in S_{d}$.

Lemma 4.10. If $X$ is a distribution design realizing $\mathcal{R}$, then $X$ realizes $\operatorname{cl}(\mathcal{R})$.

We next define $\mathcal{R}_{S}$, the projection of $\mathcal{R}$ to permutations of $S$. 
Definition 4.11. Let $\mathcal{R}$ be a set of constraints and $S \in$ $\langle n\rangle$. We define $\mathcal{R}_{S} \triangleq$

$\left\{\right.$ " $Q_{1} \circ Q_{2}$ ": " $Q_{1} \circ Q_{2}$ " $\left.\in \mathcal{R} \wedge \exists_{\pi_{1}, \pi_{2}} Q_{1}=\pi_{1}(S), Q_{2}=\pi_{2}(S)\right\}$,

that is, $\mathcal{R}_{S}$ contains all constraints in $\mathcal{R}$ in which both sequences are permutations of $S$.

ThEOREM 4.12. A d-homogeneous set of constraints $\mathcal{R}$ is realizable if and only if for every sequence $S \in\langle n\rangle_{d}$ the set of constraints $\operatorname{cl}(\mathcal{R})_{S}$ is realizable.

Theorem 4.12 provides a necessary and sufficient condition characterizing when a $d$-homogeneous set of constraints is realizable. The proof of Theorem 4.12 follows from Lemma 4.10, where we prove that the condition is necessary (since if $\mathcal{R}$ is realizable, then $\operatorname{cl}(\mathcal{R})$ and, hence, $\operatorname{cl}(\mathcal{R})_{S}$ are realizable), and a construction of a distribution design showing that the condition is sufficient, which is described in the full version of this paper.

The next theorem, whose proof appears in the full version of this paper, summarizes the properties of the distribution design for homogenous sets of constraints.

THEOREM 4.13. There is an algorithm running in time poly $(|\mathcal{R}|, n)$ that, given a d-homogeneous set of constraints $\mathcal{R}$ on $n$ variables, determines if $\mathcal{R}$ is realizable. If $\mathcal{R}$ is realizable, then there is a distribution design realizing it with share size $n^{O\left(d^{2}\right)}$.

The description of the construction of the distribution design for non-homogenous sets of constraints appears in the full version.

\section{APPLICATION TO MPC}

As discussed in the introduction, the problem of distribution design can be seen as a strict generalization of several well-studied problems in cryptography. These include secret sharing, garbling schemes, and non-interactive protocols for secure multiparty computation. We demonstrate the usefulness of more general instances of distribution design by describing an application that is not captured by any of the above primitives.

We consider an ad-hoc flavor of secure multiparty computation (MPC), where it is known in advance which function should be computed but the identity of the participants is not known a-priori. The output of the function may depend just on the inputs of the participants, or alternatively may also depend on their identities or even on the order in which they show up. That is, this function accepts as input a sequence of at most $k$ pairs of the form $\left(i, w_{i}\right)$, where the first entry is an identity of a party and the second entry is its secret input. We assume for simplicity that the parties are honest-but-curious and that each party may participate at most once. Ideally, we would like such ad-hoc MPC protocols to also guarantee anonymity, namely hide everything except the number of participating parties and the output of the function.

In the standard interactive model for MPC, the ad-hoc nature of the protocol does not pose a significant challenge. Indeed, if the set of participating parties can agree on pseudoidentities and communicate directly with each other, then they can simply use a standard general-purpose MPC protocol to evaluate the desired function on the inputs $\left(i, w_{i}\right)$.
However, as we argue below, in the case of non-interactive MPC protocols, where the single message sent by each party cannot depend on the messages of the other parties, this variant of the model introduces significant new challenges.

PSM protocols. As a baseline, consider the private simultaneous messages (PSM) model of Feige et al. [5]. In this model there are $k$ parties $P_{1}, \ldots, P_{k}$ where each party $P_{i}$ holds a secret input $w_{i} \in W$ and all parties have access to common randomness $r$. The parties wish to securely evaluate a function $f$ on their inputs by simultaneously sending messages to a referee, where the message sent by $P_{i}$ may only depend on $w_{i}$ and $r$. The referee, who does not know $r$, should be able to correctly recover $f\left(w_{1}, \ldots, w_{k}\right)$ from the $k$ messages but should learn no additional information about the inputs. Stronger notions of PSM protocols, which guarantee the best possible secrecy even when the referee colludes with subsets of parties, were considered in [2]. However, here we assume that only the referee is corrupted.

Ad-hoc PSM protocols. Suppose we would like to run a PSM protocol even if only $d<k$ parties wish to participate and send messages, where the output of $f$ may depend on the inputs of the participants and possibly also on their identities. The function $f$ is defined over sequences of inputs of the form $\left(i, w_{i}\right)$, where each $i$ is guaranteed to appear at most once in the sequence. Given an input sequence, we would like the referee to learn the value of $f$ on this sequence, but learn no additional information about the input sequence, including the identity of participants, except what should inevitably be leaked (namely, the value of $f$ on all subsequences and their permutations).

We will typically be interested in symmetric $f$, where the output is not sensitive to the order in which the inputs $\left(i, w_{i}\right)$ are given, but one may also consider non-symmetric $f$ (e.g., for giving priority to parties who act faster). It is also useful to consider partial $f$ that are only defined on sequences of bounded length.

As an example for a simple symmetric $f$, consider the following problem of "matching in the dark." There are $n$ people whose matching preferences are represented by an undirected graph (which is known to the protocol designer). When two people would like to go out, they anonymously send messages to the referee. The referee should learn whether they have mutual interest in going out together without learning anything else about their identities. In this case, the function $f$ depends only on the identities of the participating parties (i.e., the $w_{i}$ are empty) and it is defined on sequences of length at most 2. The output of $f$ on input $(i, j)$ is "yes" or "no" depending on whether $i$ and $j$ are connected, and its output on input $i$ (a sequence of length 1) is "no". If the matching criterion may also depend on the type of activity (e.g., "going to a movie" or "going to a restaurant") then the inputs $w_{i}$ can contain this additional information.

An ad-hoc PSM protocol for $f$ is defined as follows. Syntactically, such a protocol is very similar to a standard PSM protocol. As in standard PSM, each party $P_{i}$ is defined by a message function $M_{i}\left(r, w_{i}\right)$ that determines the message it sends to the referee on input $w_{i}$ and common randomness $r$. The referee is defined by a reconstruction function that given a sequence of messages produces an output. However, unlike standard PSM, here the sequences can contain anywhere from 1 to $k$ messages. The correctness requirement is that on any input sequence for which the output of $f$ is de- 
fined, the referee should reconstruct the correct output with probability 1 over the choice of $r$. The security requirement should take into account the inevitable attacks mentioned above, namely computing $f$ on sub-sequences of inputs and their permutations. Concretely, for any two input sequences of the same length such that the value of $f$ on all of their (permuted) sub-sequences is the same, the distributions of the corresponding two message sequences should be identical. See full version for a simulation-based variant of the definition.

An ad-hoc PSM protocol for $f$ can be reduced to an instance of distribution design in the following natural way. Assume for simplicity that each input $w_{i}$ is a single bit (an extension to the general case is straightforward). The design involves $n=2 k$ variables $X_{i}^{b}$, where $1 \leq i \leq k$ and $b \in\{0,1\}$, and where $X_{i}^{b}$ denotes the message of $P_{i}$ on input $w_{i}=b$. For any two sequences $I=\left(\left(i_{1}, w_{i_{1}}\right), \ldots,\left(i_{d}, w_{i_{d}}\right)\right)$ and $I^{\prime}=\left(\left(i_{1}^{\prime}, w_{i_{1}^{\prime}}^{\prime}\right), \ldots,\left(i_{d}^{\prime}, w_{i_{d}^{\prime}}^{\prime}\right)\right)$ on which $f$ has different outputs, we include a disjointness constraint between the corresponding variable sequences. If $I$ and $I^{\prime}$ satisfy the above criterion for indistinguishability, then we include an equality constraint.

Our results provide a perfectly secure ad-hoc PSM protocol for every $f$, whose complexity is generally exponential in the input length. However, we do get polynomial-time solutions in cases where the input domain is finite and the length of admissible sequences is bounded by a constant. For instance, the matching in the dark problem described above reduces to a 2-homogenous, projective distribution design. We defer further details about the application to ad-hoc PSM to the full version.

\section{DISTRIBUTION DESIGN WITH IMPLICIT CONSTRAINTS}

In this section we define distribution designs generators, in which the constraints are given in an implicit representation and the generation of the shares and the reconstruction of the equivalence class of a set from their shares should be done in polynomial time. We consider three variants of the security for distribution designs generators, computational, statistical, and perfect. The emphasis of this section is on computationally-secure distribution designs generators, where it is only required that a polynomial-time adversary cannot distinguish between shares of two equivalent sequences. We then construct computational distribution designs for projective homogeneous sets of constraints using previous constructions for multi-input functional encryption (MIFE) [9]. Finally, we show that in the general (non-projective) case, if $\mathrm{RP} \neq \mathrm{NP}$, then there is no distribution designs even for homogeneous sets of constraints.

\subsection{Definition of Distribution Designs for Im- plicit Constraints}

We first define implicit representations of a set of constraints for a distribution design by a circuit specifying the equivalence class of each sequence. This gives a much more compact representation of a set of constraints.

Definition 6.1 (Implicit REPRESEntation). An implicit representation of a set of constraints with $n$ variables is a circuit $^{1} C$ with $n \log n$ inputs and $\lceil\log (t+1)\rceil$ outputs (for some integer $t$ ) computing a function $f:\langle n\rangle \rightarrow[t] \cup\{0\}$, which represents the set of constraints

$$
\begin{aligned}
\mathcal{R}_{f}= & \{S \equiv Q: f(S)=f(Q) \neq 0\} \\
& \bigcup\{S \| Q: f(S) \neq f(Q), f(S) \neq 0, f(Q) \neq 0\} .
\end{aligned}
$$

When we represent projective constraints, the circuit gets $n$ bits as its input (i.e., a characteristic vector of a set) and it computes a function $f: 2^{[n]} \rightarrow[t] \cup\{0\}$.

In an implicit representation, we specify an equivalence class for each sequence, where 0 is a "don't care". Two sequences of the same length whose equivalence class is nonzero are equivalent if their equivalence class (i.e., the value of $f$ computed on them) are the same, and they are disjoint otherwise. Clearly, every set of constraints described by an implicit representation can be explicitly represented (usually, with a much longer description). The other direction is not true. For example, for $\mathcal{R}=\{"(1,2) \equiv(3,4)$ ", " $(1,3) \equiv$ $(2,4) "\}$, the sequences $(1,2)$ and $(1,3)$ are not constrained. This set of constraints cannot be implicitly represented.

We next define distribution designs generators for implicit constraints. We use the term generator since we have an encoding algorithm, which gets as an input a circuit representing the constraints, and generates the shares. This algorithm should run in time polynomial in the circuit size and in a security parameter.

The output of our generator is $n+1$ values $x_{0}, x_{1}, \ldots, x_{n}$. We think of $x_{0}$ as a public share. It is used to simply the presentation and to separate between public parameters and private information. Technically speaking, given a distribution design $X=\left(X_{0}, X_{1}, \ldots, X_{n}\right)$ with public information, we can define a a distribution design $X^{\prime}=\left(X_{1}^{\prime}, \ldots, X_{n}^{\prime}\right)$ without public information, where $X_{i}^{\prime}=X_{0} \circ X_{i}$; the results is a distribution designs satisfying the same constraints. ${ }^{2}$

Definition 6.2 (Distribution DESIGNS GENERATOR). $A$ distribution design generator for a class of circuits $\mathcal{C}$ consists of two algorithms (ENC, DEC) described below.

Encoding. $\operatorname{ENC}\left(1^{k}, C\right)$ is a probabilistic polynomial-time algorithm (abbreviated PPT algorithm) that takes as and input a security parameter $1^{k}$ and a circuit $C \in \mathcal{C}$ and outputs $n+1$ shares $\left(x_{0}, x_{1}, \ldots, x_{n}\right)$.

Decoding. $\operatorname{Dec}\left(x_{0}, x_{1}, \ldots, x_{d}\right)$ is a deterministic polynomial time algorithm that takes as input $d$ shares, for some $1 \leq d \leq n$, and outputs an integer in $[t]$.

$A$ distribution design generator for a class of circuits $\mathcal{C}$ is correct if for every $n$, every circuit $C \in \mathcal{C}$ representing constraints on $n$ variables, every $d \in[n]$, and every $S=$

\footnotetext{
${ }^{1}$ We can also consider other models computing the function $f$, e.g., formulas. This can change the efficiency measure of the distribution design generator, since we will alow it to run in time polynomial in the size of the representation of $f$.

${ }^{2}$ Furthermore, in the information-theoretic setting we can choose a possible value $x_{0}$ for $X_{0}$ and sample $\left(X_{0}, \ldots, X_{n}\right)$ conditioned on $X_{0}=x_{0}$. The result is a distribution design satisfying the constraints of $\mathcal{R}$ whose share size is $\max _{i \in[n]}\left\{\left|X_{i}\right|\right\}$. The above transformation, in general, does not preserve the efficiency of sampling from $\left(X_{0}, \ldots, X_{n}\right)$.
} 
$\left(i_{1}, \ldots, i_{d}\right) \in\langle n\rangle_{d}$ such that $C(S) \neq 0$ :

$$
\operatorname{Pr}\left[\begin{array}{c}
\left(x_{0}, x_{1}, \ldots, x_{n}\right) \leftarrow \operatorname{ENC}\left(1^{k}, C\right) ; \\
\operatorname{DEC}\left(x_{0}, x_{i_{1}}, \ldots, x_{i_{d}}\right)=C(S)
\end{array}\right] \geq 1-\operatorname{negl}(k),{ }^{3}
$$

where the probability is taken over the coins of ENC. Notice that there are no correctness requirement when $C(S)=0$.

Next we define the security of distribution design generators.

DeFinition 6.3. Consider the following game between an adversary $\mathcal{A}$ and a challenger:

1. The adversary on input $1^{k}$ generates a circuit $C \in \mathcal{C}$. Let $n$ be the number of variables in the constraints represented by $C$. The adversary also chooses two sequences $S_{0}=\left(i_{1}^{0}, \ldots, i_{d}^{0}\right), S_{1}=\left(i_{1}^{1}, \ldots, i_{d}^{1}\right) \in\langle n\rangle$ such that $C\left(S_{0}\right)=C\left(S_{1}\right) \neq 0$. The adversary sends $1^{k}, C$, $S_{0}, S_{1}$ to the challenger.

2. The challenger chooses with uniform distribution a bit $b \in\{0,1\}$ and sets $\left(x_{0}, x_{1}, \ldots, x_{n}\right) \leftarrow \operatorname{ENC}\left(1^{k}, C\right)$. It then sends $\left(x_{0}, x_{i_{1}^{b}}, \ldots, x_{i_{d}^{b}}\right)$ to the adversary.

3. The adversary outputs a bit $b^{\prime}$.

The adversary wins the game if $b^{\prime}=b$.

We say that a distribution design generator (ENC, DEC) is computationally secure if for every non-uniform polynomialtime adversary $\mathcal{A}$, the probability that $\mathcal{A}$ wins is at most $1 / 2+\operatorname{negl}(k)$ for some negligible function negl. Similarly, we say that a distribution design generator is statistically secure if for every unbounded adversary $\mathcal{A}$, the probability that $\mathcal{A}$ wins is at most $1 / 2+\operatorname{negl}(k)$ for some negligible function negl, and it perfectly secure if for every unbounded adversary $\mathcal{A}$, the probability that $\mathcal{A}$ wins is at most $1 / 2$.

DeFinition 6.4. A distribution design generator for projective constraints is a distribution design generator for the class of circuits representing projective constraints. The correctness and security is only required for sets $S \subseteq[n]$.

\subsection{Projective Distribution Designs Generators and Non-Interactive Multi-Party Protocols}

Protocols for non-interactive secure multi-party computation (NIMPC) were studied by Beimel et al. [2]. NIMPC protocols strengthen PSM protocols by additionally considering collusions between the referee and subsets of the parties. The focus of [2] was on perfectly secure NIMPC protocols. In this work we are interested in broader feasibility results, thus, we consider also computationally secure NIMPC protocols, in which all participants and the adversary run in polynomial time.

Similarly to PSM protocols, an NIMPC protocol involve $n$ parties $P_{1}, \ldots, P_{n}$, and a referee. (For simplicity, we restrict the attention to the case of single-bit inputs.) An NIMPC protocol additionally involves a dealer that distributes correlated randomness to the parties. The dealer's inputs are a circuit $C$, which computes a function $f:\{0,1\}^{n} \rightarrow\{0, \ldots, t\}$ for some integers $n, t$, and a security parameter $1^{k}$. It computes $2 n$ messages $\left(M_{i, \sigma}\right)_{i \in[n], \sigma \in\{0,1\}}$ and gives to $P_{i}$ the messages $M_{i, 0}, M_{i, 1}$ for every $i \in[n]$. Each party $P_{i}$, holding input $x_{i} \in\{0,1\}$, sends the message $M_{i}=M_{i, x_{i}}$ to the

\footnotetext{
${ }^{3} \mathrm{By} \operatorname{negl}(\cdot)$ we denote a negligible function, i.e., a function that vanishes faster than any (positive) inverse-polynomial.
}

referee. The referee after getting $n$ messages $M_{1}, \ldots, M_{n}$ should be able to efficiently compute $f\left(x_{1}, \ldots, x_{n}\right)$.

Notice that in such non-interactive protocols, a coalition $T \subseteq\left\{P_{1}, \ldots, P_{n}\right\}$ that hears all messages sent in the protocol can compute $f$ on many points: given messages $\left(M_{i, x_{i}}\right)_{i \notin T}$ (for unknown inputs $\left(x_{i}\right)_{i \notin T}$ ), the set $T$ can simulate the referee with messages $\left(M_{i, y_{i}}\right)_{i \in T}$ for every inputs $\left(y_{i}\right)_{i \in T}$. Informally, the protocol is said to be robust if every set $T$ can only learn this information, namely, a coalition $T$ can learn no more than the restriction of $f$ fixing the inputs of parties not in $T$.

Definition 6.5. An NIMPC protocol consists of two algorithms (NIMPCENC, NimPCDEC) described below.

Encoding. NIMPCENC $\left(1^{k}, C\right)$ is a PPT algorithm that takes as input a security parameter $1^{k}$ and a circuit $C$ and outputs $2 n$ messages $\left(M_{i, \sigma}\right)_{i \in[n], \sigma \in\{0,1\}}$.

Decoding. NimpcDeC $\left(M_{1}, \ldots, M_{n}\right)$ is a deterministic polynomial time algorithm that takes as input $n$ messages $M_{1}, \ldots, M_{n}$ and outputs an integer in $[t]$.

An NIMPC protocol is correct if for every $n$, every circuit $C$ computing a function with $n$ bit input, and every $x \in\{0,1\}^{n}$ :

$$
\begin{aligned}
\operatorname{Pr}\left[\left(M_{i, \sigma}\right)_{i \in[n], \sigma \in\{0,1\}} \leftarrow\right. & \operatorname{NimpCENC}\left(1^{k}, C\right) ; \\
& \left.\operatorname{DeC}\left(M_{1, x_{1}}, \ldots, M_{n, x_{n}}\right)=C(x)\right] \\
\geq & 1-\operatorname{negl}(k),
\end{aligned}
$$

where the probability is taken over the coins of NIMPCENC.

We next formalize the robustness requirement of NIMPC protocols.

DEFINITION 6.6. Consider the following game between an adversary $\mathcal{A}$ and a challenger:

1. The adversary on input $1^{k}$ generates a circuit $C$. Let $n$ be the number of inputs in $C$. The adversary also chooses a set $T$ and two inputs for $\bar{T}$, namely, $\left(x_{i}^{0}\right)_{i \notin T}$ and $\left(x_{i}^{1}\right)_{i \notin T}$. The adversary sends $C, T,\left(x_{i}^{0}\right)_{i \notin T},\left(x_{i}^{1}\right)_{i \notin T}$ to the challenger.

2. The challenger chooses a bit $b \in\{0,1\}$ and computes messages $\left(M_{i, \sigma}\right)_{i \in[n], \sigma \in\{0,1\}}$ for the circuit $C$. It then sends $\left(M_{i, 0}, M_{i, 1}\right)_{i \in T}$ and $\left(M_{i, x_{i}^{b}}\right)_{i \notin T}$ to the adversary.

3. The adversary outputs a bit $b^{\prime}$.

The adversary wins if $b^{\prime}=b$ and if for every $\left(y_{i}\right)_{i \in T}$

$$
f\left(\left(y_{i}\right)_{i \in T},\left(x_{i}^{0}\right)_{i \notin T}\right)=f\left(\left(y_{i}\right)_{i \in T},\left(x_{i}^{1}\right)_{i \notin T}\right) .
$$

We say that an NIMPC protocol is computationally-secure if for every non-uniform polynomial-time adversary $\mathcal{A}$, the probability that $\mathcal{A}$ wins is at most $1 / 2+\operatorname{negl}(k)$ for some negligible function negl. Similarly, we say that an NIMPC protocol is statistically-secure if for every unbounded adversary $\mathcal{A}$, the probability that $\mathcal{A}$ wins is at most $1 / 2+\operatorname{negl}(k)$ for some negligible function negl, and it is perfectly-secure if for every unbounded adversary $\mathcal{A}$, the probability that $\mathcal{A}$ wins is at most $1 / 2$.

In our security definition, an NIMPC protocol hides the information on $\left(x_{i}\right)_{i \notin T}$. The security requirement in [2] is stronger as it requires that the NIMPC protocol also hides 
the function. We can strengthen our definition by allowing the adversary to choose two circuits $C_{0}, C_{1}$ instead and the challenger computes the messages for the circuit $C_{b}$. We define the weaker notion as it suffices for constructing distribution design generators.

We next show that if there exists a secure NIMPC protocol for all circuits, then there exists a secure generator for projective homogeneous distribution designs. Our result applies to a broader class of projective distribution designs, which we define below.

Definition 6.7 (Subset CONSISTENCY). We say that an implicit representation $f$ of a set of projective constraints is subset consistent if for every two sets $A, B \subset[n]$ such that $f(A)=f(B) \neq 0$ and for every $S \subseteq[d]$, where $d=|A|=$ $|B|$,

$$
f\left(A_{S}\right)=f\left(B_{S}\right){ }^{4}
$$

We represent a projective $d$-homogeneous set of constraints by a circuit computing a function $f: 2^{[n]} \rightarrow[t]$, where $f(x)=0$ for every $x \in\{0,1\}^{n}$ whose weight is not $d$ (that is, the circuit returns "Don't care" for every set whose size is not $d$ ). Thus, projective $d$-homogeneous sets of constraints are subset consistent. Furthermore, if $C$ represents a fullyspecified projective set of constrains (i.e., $C(S) \neq 0$ for all sets $S$ ), then subset-closure is a necessary condition for realizability of the set of constraints.

THEOREM 6.8. Let type $\in\{$ computationally, statistically, perfectly\}. If there is a type-secure NIMPC protocol for all circuits, then there is a type-secure generator for subsetconsistent projective distribution designs, in particular, for homogeneous projective distribution designs.

Proof. Let (NimpcEnc, NimpcDec) be a secure NIMPC protocol. We would want to simply execute the NIMPC protocol with the circuit describing the constraints. However, this is not possible since an NIMPC protocol does not hide the identity of the party sending the message. To hide this identity, we first shuffle the order of the inputs and then execute the NIMPC protocol.

We construct a distribution design generator (ENC, DEC) as follows:

\section{The ecncoding algorithm ENC.}

- Input. A circuit $C$ computing a function $f$ (with inputs $\left.x_{1}, \ldots, x_{n}\right)$ and a security parameter $1^{k}$,

- Construct a circuit $C^{\prime}$ computing the following function $g$ with $n+1$ inputs

$$
g\left(\pi, x_{1}, \ldots, x_{n}\right) \triangleq f\left(x_{\pi(1)}, \ldots, x_{\pi(n)}\right),
$$

where $\pi$ is a permutation in $S_{n}$.

- Choose a random permutation $\rho \in S_{n}$.

- Compute NimpcEnc $\left(1^{k}, C^{\prime}\right)$ - the messages for the function $g$; denote by $M_{\rho}$ the message of the 0 -th player with input $\rho$ and, for $i \in[n]$ and $j \in\{0,1\}$, denote by $M_{i, j}$ the message of the $i$-th player with input $j$.

\footnotetext{
${ }^{4}$ A similar condition to subset consistency, namely, $f\left(A_{S}\right)=$ $f\left(B_{S}\right) \vee f\left(A_{S}\right)=0 \vee f\left(B_{S}\right)=0$, is a necessary condition for realizability of a set of requirements.
}

- Define a public share $x_{0}$ by $x_{0} \triangleq\left(M_{\rho}, M_{1,0}, \ldots, M_{n, 0}\right)$.

- For $i \in[n]$, define $x_{i} \triangleq\left(M_{\rho(i), 1}, \rho(i)\right)$.

- Output. $x_{0}, x_{1}, \ldots, x_{n}$.

The decoding algorithm DEC.

- Input. Shares $x_{0}=\left(M_{\rho}, M_{1,0}, \ldots, M_{n, 0}\right)$, $\left(x_{i}=\left(M_{j_{i}, 1}, j_{i}\right)\right)_{i \in S}$, for an unknown set $S$.

- Let $M_{j_{\ell}}=M_{j_{\ell}, 1}$ for $1 \leq \ell \leq|S|$ and $M_{j}=M_{j, 0}$ for $j \notin\left\{j_{1}, \ldots, j_{|S|}\right\}$.

- Output. $\operatorname{NimpcDec}\left(M_{\rho}, M_{1}, \ldots, M_{n}\right)$.

We first prove the correctness of ENC, DEC. For simplicity of notations, assume that $S=\{1, \ldots, d\}$, and let $y_{1}, \ldots, y_{n}$ be the characteristic vector of $S$ (that is, $y_{1}=\cdots=y_{d}=1$ and $\left.y_{d+1}=\cdots=y_{n}=0\right)$. First let $j=\rho(\ell)$ for some $1 \leq \ell \leq d$. We get that $M_{j}=M_{j, 1}=M_{j, y_{\ell}}=M_{j, y_{\rho^{-1}(j)}}$. On the other hand, for all other values of $j$ we get that $M_{j}=M_{j, 0}=M_{j, \rho^{-1}(j)}$. Thus, NimPCDEC gets the messages $M_{\rho}, M_{1, y_{\rho^{-1}(1)}}, \ldots, M_{n, y_{\rho^{-1}(n)}}$ and outputs

$$
\begin{gathered}
\operatorname{NimpcDec}\left(M_{\rho}, M_{1}, \ldots, M_{n}\right)=g\left(\rho, y_{\rho^{-1}(1)}, \ldots, y_{\rho^{-1}(n)}\right) \\
=f\left(y_{1}, \ldots, y_{n}\right)
\end{gathered}
$$

as required.

We next prove robustness. Assume towards contradiction that there is an adversary $\mathcal{A}$ that violates the security of the distribution design. We construct an adversary $\mathcal{B}$ that violates the security of the NIMPC protocol. The adversary $\mathcal{B}$ behaves as follows:

- Execute $\mathcal{A}$ and get a circuit $C$ computing a function $f$ representing some set of constraints for a distribution design and two sets $S_{0}=\left\{i_{1}^{0}, i_{2}^{0}, \cdots, i_{d}^{0}\right\}$ and $S_{1}=\left\{i_{1}^{1}, i_{2}^{1}, \cdots, i_{d}^{1}\right\}$ (where $i_{1}^{j}<i_{2}^{j}<\cdots<i_{d}^{j}$ for $j \in\{0,1\})$; let $y_{1}^{0}, \ldots, y_{n}^{0}$ and $y_{1}^{1}, \ldots, y_{n}^{1}$ be the characteristic vectors of $S_{0}$ and $S_{1}$ respectively.

- Construct a circuit $C^{\prime}$ computing the function $g$.

- Choose two random permutations $\rho_{0}$ and $\rho_{1}$ such that $\rho_{0}\left(i_{j}^{0}\right)=\rho_{1}\left(i_{j}^{1}\right) \triangleq a_{j}$ for $1 \leq j \leq d$; Set $T=\left\{a_{1}, \ldots, a_{d}\right\}$ and define $x_{\ell}^{0}=x_{\ell}^{1}=1$ if $\ell \in T$ and $x_{\ell}^{0}=x_{\ell}^{1}=0$ otherwise.

- Send $C^{\prime}, T,\left(\rho_{0},\left(x_{i}^{0}\right)_{i \notin T}\right),\left(\rho_{1},\left(x_{i}^{1}\right)_{i \notin T}\right)$ to the challenger of the NIMPC game.

- The challenger returns messages $\left(M_{\ell, 0}, M_{\ell, 1}\right)_{\ell \in T}, M_{\rho_{b}}$, $\left(M_{\ell, 0}\right)_{\ell \in[n] \backslash T}$ for a random $b$.

- Set $X_{0}=\left(M_{\rho_{b}}, M_{1,0}, \ldots, M_{n, 0}\right)$ and $Y_{j}=\left(M_{j, 1}, a_{j}\right)$ for $j \in[d]$.

- Give $X_{0}, Y_{1}, \ldots, Y_{d}$ to $\mathcal{A}$, get a bit $b^{\prime}$ from $\mathcal{A}$, and output $b^{\prime}$.

Note that in the distribution design realizing $C$, the share $X_{i_{j}^{b}}$ is $\left(M_{\rho_{b}\left(i_{j}^{b}\right)}, i_{j}^{b}\right)=\left(M_{j, 1}, a_{j}\right)$ as $\rho_{b}\left(i_{j}^{b}\right)=a_{j}$ (and $\rho_{b}$ is chosen at random). Thus, $\mathcal{A}$ gets shares of $S_{b}$ for a uniformly chosen $b$ and it returns $b^{\prime}=b$ with probability noticeably greater than $1 / 2$, and thus, $\mathcal{B}$ returns $b^{\prime}=b$ with probability noticeably greater than $1 / 2$. To complete the 
proof that this violates the security of the NIMPC protocol, we need to show that $g$ restricted to the two inputs is the same function. Formally, for $j \in\{0,1\}$ we define $g_{j}\left(\left(y_{i}\right)_{i \in T}\right)=g\left(\rho_{j},\left(x_{i}\right)_{i \notin T},\left(y_{i}\right)_{i \in T}\right)$, where $x_{i}=0$ for every $j \notin T$, and prove that $g_{0}=g_{1}$. First note that $g_{0}(1, \ldots, 1)=$ $f\left(S_{0}\right)=f\left(S_{1}\right)=g_{1}(1, \ldots, 1)$ by the choice of $\rho_{0}, \rho_{1}$ and the definition of $g$. Furthermore, since $f$ is subset consistent, $f\left(\left(S_{0}\right)_{A}\right)=f\left(\left(S_{1}\right)_{A}\right)$, thus $g_{0}\left(\left(y_{i}\right)_{i \in T}\right)=g_{1}\left(\left(y_{i}\right)_{i \in T}\right)$.

To conclude, given an adversary $\mathcal{A}$ violating the security of the generator for distribution designs, we constructed an adversary $\mathcal{B}$ violating the security of the NIMPC protocol, where both adversaries are of the same type. Assuming that the NIMPC protocol is secure, no such $\mathcal{B}$ exists, thus, the generator for distribution designs is secure.

REMARK 6.9. In the above proof, given a function $f$ with $n$ variables, we defined a function $g$ with $n+1$ variables $\rho, x_{1}, \ldots, x_{n}$, where $\rho$ is a permutation and it is not binary as we defined NIMPC protocols. To fix this, we consider $g$ as a function of $n \log n+n$ binary variables, where the first $n \log n$ variables describe $\rho$.

Goldwasser et al. [9] defined and studied multi-input functional encryption schemes (MIFE). They proved that such 1-selective secure schemes exist assuming that indistinguishability obfuscation for circuits and one-way functions exist. ${ }^{5}$ We observe that 1-selective multi-input functional encryption schemes can be used to construct robust NIMPC protocols. A formal definition of MIFE and a proof of the following theorem appears in the full version.

THEOREM 6.10. If 1-selective multi-input functional encryption schemes for circuits exist, then computationally secure NIMPC protocols for circuits exist.

COROLlary 6.11. If indistinguishability obfuscation for circuits and one-way functions exist, then computationallysecure distribution design generators for subset-consistent sets of projective designs exist.

\subsection{Hardness Results for General Distribution Design Generators}

We next argue that, in the general case, there are no efficient distribution design generators even for homogenous sets of constraints unless $\mathrm{RP}=\mathrm{NP}$.

THEOREM 6.12. If $\mathrm{RP} \neq \mathrm{NP}$, then computationally-secure distribution design generators for circuits representing $n$ homogeneous sets of constraints over $n$ variables do not exist.

The idea of the proof is as follows. Let $f$ be such that $f(S)=0$ for every even permutation. We now plant an additional secret constraint of the form $f(S)=b$, where $S$ is a "secret" odd permutation and $b$ is either 1 or 2 . We claim that from $f$ it is hard to know if $b=1$ or $b=2$, while it would be easy to compute $b$ from a distribution design realizing $f$ generated by an efficient generator, contradicting the existence of the generator. Formally, we prove hardness via a reduction from the following hard problems.

The promise problem Ternary Unique SAT (TUSAT).

${ }^{5}$ In contrast, the known construction provided the stronger notion of IND-secure MIFE (where the adversary chooses its messages after seeing the keys) require indistinguishability obfuscation for circuits and one-way functions that are secure against exponential-time adversaries.
Input: A boolean circuit $C:\{0,1\}^{n} \rightarrow\{0,1\}^{2}$ with the promise that there is exactly one special input on which the output is not 00 .

Output: Decide whether the output on this special input is 01 .

\section{The promise problem Unique SAT (USAT).}

Input: A boolean circuit $F:\{0,1\}^{n} \rightarrow\{0,1\}$ with the promise that $F$ has at most one satisfying assignment.

Output: Decide whether $F$ is satisfiable or not.

Claim 6.13. If TUSAT $\in \mathrm{BPP}$, then $\mathrm{RP}=\mathrm{NP}$.

Proof. By [15], if USAT $\in \mathrm{BPP}$, then $\mathrm{RP}=\mathrm{NP}$. We show that if TUSAT $\in$ BPP then USAT $\in$ BPP. Without loss of generality, we assume that there exists a probabilistic polynomial-time algorithm $\mathcal{A}$ for TUSAT, whose error is less than $1 / 4 n$. We construct a randomized algorithm $\mathcal{A}^{\prime}$ for USAT, whose (one-sided) error is less than $1 / 4$. Given a USAT instance $F$ with exactly one satisfying assignment, we can extract this assignment using $\mathcal{A}$ as we next describe. For every $i \in[n]$, construct a circuit $C_{i}$, where $C_{i}(x)=\left(\overline{x_{i}} \wedge F(x), x_{i} \wedge F(x)\right)$ (that is, $C_{i}(x)$ outputs 00 if $F(x)=0$ and otherwise its output is 01 or 10 depending on the $i$-th bit of $x$ ) and execute $\mathcal{A}$ on $C_{i}$. If $C_{i} \in$ TUSAT, set $a_{i}=1$; otherwise set $a_{i}=0$. Finally, if $F\left(a_{1}, \ldots, a_{n}\right)=1$, answer "YES", otherwise answer "NO". If $F$ is unsatisfiable, then, clearly, $F\left(a_{1}, \ldots, a_{n}\right)=0$, and $\mathcal{A}^{\prime}$ answers "NO". On the other hand, assume that $F$ has a unique satisfying assignment $\left(a_{1}, \ldots, a_{n}\right)$. If $a_{i}=0$ then $C_{i}\left(a_{1}, \ldots, a_{n}\right)=10$ and $C_{i} \notin$ TUSAT. If $a_{i}=1$ then $C_{i}\left(a_{1}, \ldots, a_{n}\right)=01$ and $C_{i} \in$ TUSAT. To conclude, the probability that $\mathcal{A}$ errs in one of its $n$ executions is less than $1 / 4$, and if it does not err, $\mathcal{A}^{\prime}$ returns the correct answer for the promise problem USAT.

We now argue that an efficient computationally-secure distribution design generator for non-homogenous sets of constraints implies a probabilistic polynomial-time algorithm for TUSAT (the same hardness result holds for statisticallysecure and perfect distribution design generators).

LEMMA 6.14. If there is a computationally-secure distribution design generator for $n$-homogeneous sets of constraints over $n$ variables, then TUSAT $\in$ BPP.

Proof. Given a TUSAT instance $C$ with input length $m$, define an implicit representation $f$ of an $n$-homogeneous set of constraints for a distribution design with $n=3 m+2$ variables as follows. First, if $S$ is an even permutation then $f(S)=2$ (note that determining if $S$ is an even permutation can be done efficiently). Next, for every $x \in\{0,1\}^{m}$ we define an odd permutation $S_{x}$ as follows: $S_{x}(1)=2, S_{x}(2)=$ 1 and for every $i \in[m]$ if $x_{i}=1$ then $S_{x}(3 i)=3 i+1, S_{x}(3 i+$ $1)=3 i+2, S_{x}(3 i+2)=3 i$, else $S_{x}(3 i)=3 i, S_{x}(3 i+1)=$ $3 i+1, S_{x}(3 i+2)=3 i+2$. That is, $S_{x}$ is composed of one transposition between 1 and 2 , and from 3 cycles for every $i$ such that $x_{i}=1$; hence $S_{x}$ is an odd permutation. Let $f\left(S_{x}\right)=0$ if $C(x)=00, f\left(S_{x}\right)=2$ if $C(x)=01$, and $f\left(S_{x}\right)=1$ if $C(x) \in\{10,11\}$. Otherwise (that is, if $S$ is a permutation not in the above forms or if the length of $S$ is less than $n$ ), let $f(S)=0$. The above construction implies that the special input for $C$ encodes an odd permutation 
on which the output of $f$ is either 1 or 2 depending on the output of $C$ on this special input. Furthermore, $f$ can be computed by a circuit whose size is polynomial in the size of the original size of $C$ and in $m$.

We now describe a probabilistic polynomial-time algorithm $\mathcal{B}$ for TUSAT(assuming a distribution design generator), whose input is a circuit $C$ satisfying the promise of TUSAT.

- Construct a circuit $F$ computing the constraints for the function $f$ described above.

- Let $\left(x_{1}, \ldots, x_{3 m+2}\right) \leftarrow \operatorname{ENC}\left(1^{n}, F\right)$.

- If $\operatorname{DEC}\left(x_{2}, x_{1}, x_{3}, \ldots, x_{3 m+2}\right)=2$, then output "YES", else output "NO".

Assume that $\mathcal{B}$ errs with probability at least $1 / 4$ on infinite number of inputs satisfying the TUSAT promise. Let $C_{1}, C_{2}, \ldots$ be an infinite sequence of inputs on which $\mathcal{B}$ errs, where the size of $C_{i}$ is $s_{i}$ and $s_{1}<s_{2}<\ldots$ Let $m_{i}$ be the number of variables in $C_{i}$. Furthermore, let $a^{i}$ be the special input of $C_{i}$ for $i \in \mathbb{N}$. We now construct a non-uniform adversary violating the security of the distribution design generator. On input $1^{k}$, the adversary outputs $C_{i}$ for the largest $i$ such that $s_{i}<k$, and the sequences $\left(1,2, \ldots, 3 m_{i}+2\right)$ and $S_{a_{i}}^{\prime}$, where $S_{a_{i}}^{\prime}(j)=j$ for $j \in\{1,2\}$ and $S_{a_{i}}^{\prime}(j)=S_{a_{i}}(j)$ for $j \in\left\{3, \ldots, 3 m_{i}+2\right\}$ (notice that both of these permutations are even). Let $\left(y_{1}, \ldots, y_{3 m_{i}+2}\right)$ be the shares that the adversary gets from the challenger and $d \leftarrow \operatorname{DEC}\left(y_{2}, y_{1}, y_{3}, \ldots, y_{3 m_{i}+2}\right)$. Now, if $C_{i} \in$ TUSAT, then the adversary returns $b^{\prime}=3-d$, otherwise it returns $b^{\prime}=-d+2$. We claim that the adversary wins with probability nearly $3 / 4$.

First, assume that $C_{i} \in$ TUSAT, hence, $f\left(S_{a^{i}}\right)=2$. Thus, by the correctness of the distribution design, if $b=1$, then $d=\operatorname{DEC}\left(y_{2}, y_{1}, y_{3}, \ldots, y_{3 m_{i}+2}\right)=2$ with probability $1-\operatorname{negl}(n)$. However, since $\mathcal{B}$ errs on $C_{i}$ with probability at least $1 / 4$, if $b=0$, then $\operatorname{DEC}\left(y_{2}, y_{1}, y_{3}, \ldots, y_{3 n_{i}+2}\right)=$ $\operatorname{DEC}\left(x_{2}, x_{1}, x_{3}, \ldots, x_{3 m_{i}+2}\right) \neq 2$, with probability at least $1 / 4$. Thus, the adversary can distinguish between the two permutations by computing $\operatorname{DEC}\left(y_{2}, y_{1}, y_{3}, \ldots, y_{3 m_{i}+2}\right)$, although both permutations are even, thus, should be indistinguishable. Similar arguments hold when $C_{i} \notin$ TUSAT. This implies that (assuming the existence of a secure distribution design generator), algorithm $\mathcal{B}$ can only err with probability at least $1 / 4$ on a finite number of inputs.

Acknowledgements. This work was done in part while the first, third, and forth authors were visiting the Simons Institute for the Theory of Computing, supported by the Simons Foundation and by the DIMACS/Simons Collaboration in Cryptography through NSF grant \#CNS-1523467. Research by the first three authors received funding from the European Union's Tenth Framework Programme (FP10/20102016) under grant agreement no. 259426 ERC-CaC. The first author was supported by ISF grant $544 / 13$ and by the Frankel center for computer science. Research by the second author received funding from the European Union's Seventh Framework Programme (FP7/2007-2013) under grant agreement no. 257575. Research by the third author received funding from a DARPA/ ARL SAFEWARE award, NSF Frontier Award 1413955, NSF grants 1228984, 1136174, 1118096, and 1065276. This material is based upon work supported by the Defense Advanced Research Projects Agency through the ARL under Contract W911NF-15-C-0205. The views expressed are those of the author and do not reflect the official policy or position of the Department of Defense, the National Science Foundation, or the U.S. Government. The third and fourth authors were supported by ISF grant 1709/14 and BSF grant 2012378.

\section{REFERENCES}

[1] B. Barak, O. Goldreich, R. Impagliazzo, S. Rudich, A. Sahai, S. P. Vadhan, and K. Yang. On the (im)possibility of obfuscating programs. J. of the ACM, 59(2):6, 2012.

[2] A. Beimel, A. Gabizon, Y. Ishai, E. Kushilevitz, S. Meldgaard, and A. Paskin-Cherniavsky. Non-interactive secure multiparty computation. In CRYPTO 2014, vol. 8617 of LNCS, pp. 387-404, 2014.

[3] M. Bellare, V. T. Hoang, and P. Rogaway. Foundations of garbled circuits. In CCS '12, pages 784-796, 2012.

[4] G. R. Blakley. Safeguarding cryptographic keys. In Proc. of the 1979 AFIPS National Computer Conference, volume 48, pages 313-317, 1979.

[5] U. Feige, J. Kilian, and M. Naor. A minimal model for secure computation. In Proc. of the 26th STOC, pages 554-563, 1994.

[6] M. L. Furst, J. E. Hopcroft, and E. M. Luks. Polynomial-time algorithms for permutation groups. In Proc. of the 21st FOCS, pages 36-41, 1980.

[7] S. Garg, C. Gentry, S. Halevi, M. Raykova, A. Sahai, and B. Waters. Candidate indistinguishability obfuscation and functional encryption for all circuits. In Proc. of the 54th FOCS, pages 40-49, 2013.

[8] O. Goldreich, S. Micali, and A. Wigderson. How to play any mental game. In Proc. of the 19th STOC, pages 218-229, 1987.

[9] S. Goldwasser, S. D. Gordon, V. Goyal, A. Jain, J. Katz, F. Liu, A. Sahai, E. Shi, and H. Zhou. Multi-input functional encryption. In EUROCRYPT 2014, volume 8441 of $L N C S$, pages 578-602, 2014.

[10] Y. Ishai and E. Kushilevitz. Perfect constant-round secure computation via perfect randomizing polynomials. In Proc. of the 29th ICALP, volume 2380 of $L N C S$, pages 244-256, 2002.

[11] I. Komargodski and M. Zhandry. Cutting-edge cryptography through the lens of secret sharing. IACR Cryptology ePrint Archive, 2015:735, 2015. To appear in Proc. of TCC 2016A.

[12] A. Seress. Permutation Group Algorithms, volume 152 of Cambridge Tracts in Mathematics. Cambridge University Press, 2003.

[13] A. Shamir. How to share a secret. Communications of the ACM, 22:612-613, 1979.

[14] C. C. Sims. Computational methods in the study of permutation groups. In Computational Problems in Abstract Algebra (Proc. Conf., Oxford, 1967), pages 169-183, 1970.

[15] L. G. Valiant and V. V. Vazirani. NP is as easy as detecting unique solutions. Theoretical Computer Science, 47:85-93, 1986.

[16] A. C. Yao. How to generate and exchange secrets. In Proc. of the 27th FOCS, pages 162-167, 1986. 